\title{
Editorial zum Themenschwerpunkt der Fachzeitschrift UmweltWirtschaftsForum (UWF)
}

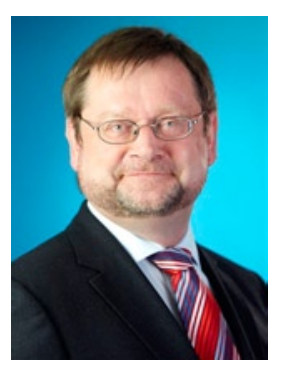

Jürgen Becker

Sehr geehrte Leserinnen und Leser,

das Potential für mehr Klimaschutz und Energieeffizienz in deutschen Unternehmen ist riesig. Bereits heute sind viele rentable Technologien vorhanden, mit denen Energiekosten eingespart, Innovationen angestoßen, die Wettbewerbsfähigkeit gestärkt und Treibhausgas-Emissionen vermindert werden können. Was wir brauchen, sind die richtigen Instrumente, mit denen die bisher brachliegenden Effizienzpotentiale in den Unternehmen endlich gehoben werden können.

Diese themenorientierte Spezial-Ausgabe beschreibt ein neues klima- und energiepolitisches Instrument, das nicht in den traditionellen Kanon staatlicher Eingriffe oder Rahmensetzungen passt. Es orientiert sich nicht an klassischen ökonomischen Modellvorstellungen, auch wenn dieses neue Instrument die Unternehmen der mittelständischen Wirtschaft als Akteure in den Fokus des Handelns rückt. Es geht bei diesem Instrument nicht um mehr Wettbewerb, sondern um mehr Kooperation und Selbstorganisation zwischen Unternehmen in einer Region oder einer Stadt. Es ist zudem ein aussichtsreicher Beitrag zur und zugleich Konkretisierung der klimapolitischen Maxime: „Global denken, lokal handeln“. Die Rede ist vom Instrument der Energieeffizienz- und Klimaschutz-Netzwerke.
Was verbirgt sich hinter diesen Energieeffizienz- und Klimaschutz-Netzwerken? Durch einen regelmäßigen und moderierten Erfahrungsaustausch zwischen 10 bis 15 Unternehmen einer Region werden in bemerkenswertem Umfang Energieeffizienz- und $\mathrm{CO}_{2}$-Minderungs-Potentiale realisiert. Dies ist nicht nur auf die reichen technischen und betriebswirtschaftlichen Kenntnisse und Erfahrungen der teilnehmenden Unternehmen zurückzuführen. Auch sozialpsychologische Zusammenhänge (z. B. die Anerkennung in der regionalen Öffentlichkeit oder in der Branche, Imageverbesserungen mit entsprechenden Marketingeffekten) und das steigende Interesse von Kunden an Themen wie Nachhaltigkeit und Ressourcenschonung tragen zu diesen vorher nicht vermuteten Erfolgen bei.

Der Weg führt dabei von einer standardisierten Initialberatung mit Maßnahmenlisten und mehrjährigen Energieeffizienz- und $\mathrm{CO}_{2}$-Minderungszielen, hin $\mathrm{zu}$ einem gemeinsamen Ziel des Unternehmens-Netzwerkes, das sich für mindestens drei, vier Jahre zu dem gemeinsamen Erfahrungsaustausch verpflichtet. Hinzu kommt ein jährliches Monitoring, das überprüft, ob der Zielpfad für das Netzwerk eingehalten wird.

Inzwischen gibt es rund 50 solcher Netzwerke in Deutschland. In der Schweiz, wo Mitte der 1980er Jahre die ersten Netzwerke entstanden, sind es bereits 70 . Der energietechnische Fortschritt dieser Netzwerke verläuft etwa doppelt so schnell wie der Fortschritt des Durchschnitts der Industrie. Auch die Energiekosten der teilnehmenden Unternehmen sinken folglich etwa doppelt so schnell.

Auch deshalb fördert das Bundesministerium für Umwelt, Naturschutz und Reaktorsicherheit im Rahmen der Nationalen Klimaschutzinitiative mit dem Projekt 30 Pilot-Netzwerke maximal 30 Unternehmens-Netzwerke mit etwa 400 Betrieben. Ziel des Projektes ist es, das Know how des Netzwerkmanagements bundesweit zu verbreiten 
und mittelfristig ein Potential von 700 Netzwerken für die mittelständische Wirtschaft zu realisieren.

Über die Funktionsweise dieses neuen Instruments der Selbstorganisation der Wirtschaft, den Energieeffizienz- und Klimaschutz-Netzwerken, deren Wirkungen und die dahinter liegenden Wirkungszusammenhänge gibt diese SpezialAusgabe einen ersten Überblick.
Ich wünsche Ihnen viel Freude bei der Lektüre und den Netzwerken auch künftig viel Erfolg.

Ihr Jürgen Becker

Staatssekretär im Bundesministerium für Umwelt, Naturschutz und Reaktorsicherheit (BMU), Berlin, Deutschland 